\title{
BMJ Open Development of a core outcome set for studies on prevention and management of pregnancy-associated venous thromboembolism (COSPVenTE): a study protocol
}

\author{
Alexandria King, ${ }^{1}$ Rohan D’Souza (D) , ${ }^{1,2,3}$ Lizabeth Teshler, ${ }^{4}$ Nadine Shehata (D) , ${ }^{5,6}$ \\ Ann K Malinowski ${ }^{1,2,3}$
}

To cite: King A, D'Souza R, Teshler L, et al. Development of a core outcome set for studies on prevention and management of pregnancy-associated venous thromboembolism (COSPVenTE): a study protocol. BMJ Open 2020;10:e034017. doi:10.1136/ bmjopen-2019-034017

- Prepublication history and additional material for this paper are available online. To view these files, please visit the journal online (http://dx.doi. org/10.1136/bmjopen-2019034017).

Received 02 September 2019 Revised 08 February 2020 Accepted 28 April 2020

D Check for updates

(c) Author(s) (or their employer(s)) 2020. Re-use permitted under CC BY-NC. No commercial re-use. See rights and permissions. Published by BMJ.

For numbered affiliations see end of article.

\section{Correspondence to} Dr Ann K Malinowski; Ann.Malinowski@ sinaihealthsystem.ca

\section{ABSTRACT}

Introduction Pregnancy and post partum are times of heightened risk for the development of venous thromboembolism (VTE), which in turn is one of the leading causes of maternal mortality and long-term morbidity. The current research aimed at improving health guidelines for women with pregnancy-associated VTE is limited by inconsistency in outcome reporting preventing comparison across studies, and lack of input from patients with respect to outcomes they propose are most important to measure. A suggested solution is the development of a core outcome set (COS) that defines the minimum criteria for outcome reporting in clinical trials and prospective studies. COSs function to facilitate data harmonisation and increase homogeneity in outcome reporting while incorporating the voice of women in this population in the planning of research to inform their ongoing care.

Methods and analysis The development of a COS for studies on pregnancy-associated VTE will comprise five steps. First, a systematic review of the published literature will identify currently reported outcomes, their definitions and measurements if applicable. This will be followed by in-person interviews with patients, clinicians, researchers, hospital administrators and policy-makers to identify outcomes they consider important. Third, the long list of outcomes obtained from steps I and II will be condensed through online Delphi surveys involving an international group of relevant stakeholders including patients. This will be followed by a face-to-face consensus meeting with representatives of all stakeholder groups to arrive at a consensus on the final COS. Lastly, to determine how the identified core outcomes should be measured, another literature review and Delphi process will be carried out as necessary.

Ethics and dissemination This study has been approved by the Mount Sinai Hospital Research Ethics Board (REB 18-0314-E). Study results will be published in openaccess journals and presented at obstetrics, maternalfetal medicine and haematology conferences. All progress will be documented on the international prospective register of systematic reviews (PROSPER0) and Core Outcome Measures in Effectiveness Trials databases. PROSPERO registration number CRD42019111479.
Strengths and limitations of this study

- Inclusion of primary and secondary outcomes reported in all experimental and observational studies, as well as in clinical trial registrations, will ensure every outcome recorded in the published and proposed literature is considered while conducting the systematic review.

- Although interviews with patients and healthcare providers will identify novel outcomes not obtained from the systematic review, getting global representation for this step might be challenging.

- Inclusion of patient stakeholders at multiple stages of core outcome set (COS) development (personal interviews for identification of outcomes, Delphi survey and consensus meeting) will ensure that patient-reported outcomes are judiciously considered for inclusion in the final COS.

- The two-round international e-Delphi survey, wherein measures are in place to maximise response rates and minimise attrition, will ensure global representation of a variety of stakeholders in the development of the final COS.

- This COS is being developed as part of the Outcome Reporting in Obstetric Studies project (https://www. obgyn.utoronto.ca/oros-project), whose investigators not only have the requisite methodological expertise but are also currently setting standards for outcome reporting in obstetric studies.

\section{INTRODUCTION}

Physiological changes affecting the haematological system during pregnancy and post partum place women at risk for the development of venous thromboembolism (VTE), including pulmonary embolism (PE) and deep vein thrombosis (DVT). ${ }^{1}$ VTE occurs at a rate of 5.4/10 000 pregnancies antepartum, 7.2/10 000 intrapartum and 4.3/10 000 post partum. ${ }^{2}$ In the USA, PE was one of the leading causes of maternal mortality, 


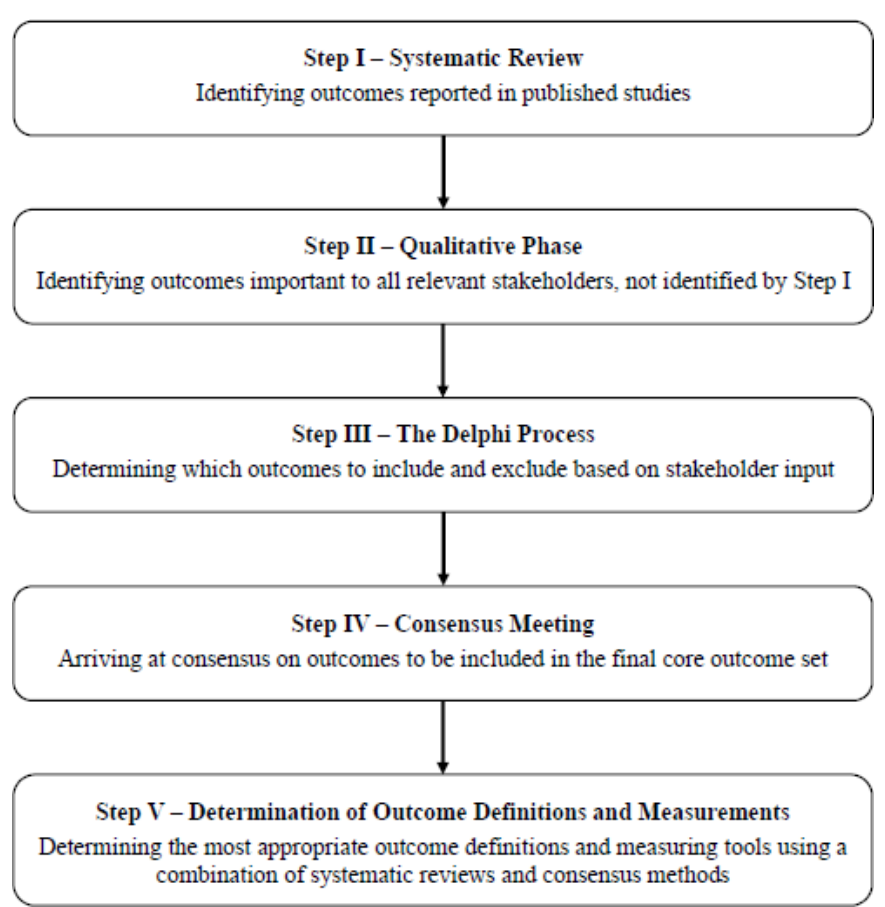

Figure 1 Steps in the development of a core outcome set.

accounting for $9 \%$ of maternal deaths between 2011 and $2014,{ }^{3}$ whereas in Canada, maternal deaths due to PE are thought to be under-reported. ${ }^{4}$ VTE is also associated with long-term morbidity in the form of post-thrombotic syndrome (PTS $)^{5}$ - with $20 \%-50 \%$ of women with lower extremity DVT demonstrating its mild form and 5\%-10\% experiencing severe symptoms. ${ }^{6-9}$ Women with PTS have reported poorer health, increased pain, skin issues and psychiatric troubles, which brought on higher frequencies of sick leave as compared with those without PTS. ${ }^{10}$

Studies addressing pregnancy-associated VTE frequently lack the methodological quality and rigour to conclusively present evidence-based recommendations to guide care. ${ }^{11-14}$ This is in part driven by the fact that pregnancy-associated VTE is fortunately quite infrequent in occurrence, yielding small sample sizes even in tertiary level institutions, lacking the necessary power to draw meaningful answers. It is compounded by heterogeneity in outcome reporting across studies, ${ }^{15}$ precluding rigorous data synthesis ${ }^{16}$ and diminishing the confidence in the available conclusions to guide practice. ${ }^{17-21}$ Furthermore, there has been a lack of patient perspective in deciding which outcomes are relevant and important to measure. ${ }^{22}$

The development of core outcome sets (COSs), a uniform/minimum set of outcomes that must be reported in all studies within a clinical area, has been proposed as a solution to heterogeneity encountered in outcome reporting. ${ }^{23} \mathrm{COS}$ development is promoted by international organisations and funding bodies, ${ }^{24} 25$ including the Core Outcome Measures for Effectiveness Trials (COMET) ${ }^{24}$ and CoRe Outcomes in Women's and Newborn health (CROWN) ${ }^{26}$ Although COSs address the issues of heterogeneity in outcome reporting to enable meta-analysis and inform clinical practice, they also function to involve the opinions of multiple stakeholders, including patients, in determining which outcomes should be reported, ensuring that all who are impacted have a voice ${ }^{2327}$ Obtaining patient and stakeholder input on health outcomes can be relatively straightforward in some conditions; however, high-risk pregnancies such as those affected by VTE pose a unique challenge-the presence of two patients (mother and fetus) whose interests can compete. ${ }^{28}$ Prioritisation of health outcomes in highrisk pregnancies therefore often involves making tradeoffs between maternal and fetal health, shown in earlier research to differ between pregnant women and other stakeholders, ${ }^{29}$ further highlighting the integral need for patient involvement in COS development. In turn, the inclusion of COS in future research has the potential to decrease research waste while allowing stronger conclusions to improve the health of women and infants. ${ }^{30}$

Thus, the objective of this protocol is to facilitate the development of a COS for studies addressing pregnancyassociated VTE (COSPVenTE), for use in relevant future trials and observational studies. Outcomes relevant to studies of prevention of VTE will be listed separately from those on management. Once used in future studies, COSPVenTE will allow researchers to form confident conclusions, eventually translating into improved prevention and management options for women with pregnancyassociated VTE.

\section{METHODS AND ANALYSIS}

As with other COS developed as part of the Outcome Reporting in Obstetric Studies project (https://www. obgyn.utoronto.ca/oros-project), the development of COSPVenTE will include five steps as shown in figure $1 .^{31}$ The development of COSPVenTE will be guided by the COMET Handbook, ${ }^{23}$ the COS-STAndards for Development $^{32}$ and the Core Outcome Set-STAndardised Protocol Items Statement, ${ }^{33}$ with adaptations specific to the scope of this project (online supplementary appendix A). The COSPVenTE protocol has been registered on the COMET website. ${ }^{34}$

COSPVenTE will comprise two distinct lists of outcomes-one for studies on prevention and the other for studies on the management of pregnancy-associated VTE as the outcomes including the use of prophylactic doses of anticoagulants in studies on prevention might defer from those including the use of therapeutic doses in studies on management.

\section{Step I: systematic review}

A systematic review of the published literature will be conducted in accordance with the Preferred Reporting Outcomes for Systematic Reviews and Meta-Analyses guidelines ${ }^{35}$ to identify numerous existing outcomes from published studies addressing the prevention and management of pregnancy-associated VTE. The protocol has been published on the international prospective register 
of systematic reviews (PROSPERO). ${ }^{36}$ The primary research question is: what are the reported outcomes in studies involving the prevention and management of VTE in women who are pregnant and up to 6 weeks post partum? The objective of the systematic review is to catalogue the outcomes currently reported in the literature that traditionally reflect aspects of the condition considered important by researchers. Components unique to the conduct of a systematic review for COS development are outlined as follow.

\section{Study selection}

MEDLINE, EMBASE, the Cochrane Central Register of Controlled Trials (CENTRAL) the Cochrane Database of Systematic Reviews, Web of Science, Scopus, CINAHL, ClinicalTrials.gov, International Clinical Trials Registry Platform and International Standard Randomized Controlled Trial Number Registry will be searched using the OVidSP platform with MeSH terms and keywords related to pregnancyassociated VTE. All clinical trials, trial registrations and observational studies with 10 or more participants will be included. This will ensure alignment with the overarching aim of COS to standardise outcome reporting in clinical trials, but will also include representation from observational studies, as trials in this population are not always feasible or ethically allowable. The inclusion of clinical trial registrations will ensure that outcomes being considered in ongoing research are also included.

\section{Data extraction and synthesis}

Data will be extracted into a standardised case report form. All reported primary and secondary outcomes, including composite outcomes will be listed. A composite outcome comprises a number of constituent outcomes, such that if one of the constituent outcomes occurs, then the composite outcome is considered to be present. ${ }^{37}$ Definitions and measurement methods, as reported by the authors of each original study, will be recorded. The purpose of this systematic review is to list all outcomes reported in the published and proposed literature, including outcomes from studies that would otherwise be considered 'low-quality' or 'at high risk-of-bias', based on methodological quality assessment. Thus, as the primary intention is outcome reporting, a methodological quality assessment will not be conducted. ${ }^{38-40}$

\section{Analysis and presentation of results}

Outcomes, their definitions and measurement tools will be listed in table form. The proportion of studies reporting each outcome and domain will be provided. Studies that did not report outcomes clearly or in a reproducible way will also be recorded. The list of outcomes obtained through the systematic review will be categorised into domains in accordance with a published taxonomy for outcomes in medical research. ${ }^{41}$

\section{Step II: stakeholder interviews}

The list of outcomes generated via systematic review is insufficient to guide COS development, as it solely reflects outcomes considered relevant by researchers. To account for outcomes deemed important by other relevant stakeholder groups, notably the patients themselves, individual interviews with women who are at risk for or have experienced VTE during pregnancy or 6 weeks post partum, as well as healthcare professionals involved in their care, will be conducted. Given the competing interests of the two individuals within the mother-fetus dyad previously identified by our group, ${ }^{28}$ this technique allows for the exploration of how trade-offs between maternal and fetal health are made as outcomes are prioritised and how decisions differ between pregnant women and other stakeholders. Further, it allows us to learn the views of stakeholders and translate their health values to reportable outcomes ${ }^{42}$ while exploring the rationale behind their choices and the influences of their social lives. ${ }^{43}$

\section{Sampling}

To ensure that the views of patients with different experiences and backgrounds are represented, specific criteria will be selected to provide maximum variation. Convenience sampling will be used to recruit patients from the Hematologic Disorders in Pregnancy Clinic at Mount Sinai Hospital who are at risk for or have experienced pregnancy-associated VTE. Healthcare providers and researchers will be recruited through email using a study flyer via contact lists assembled by the study investigators. Purposeful sampling ${ }^{44}$ will help recruit more stakeholders through known professional contacts. Participants must meet the following inclusion criteria: willingness to participate in a semistructured, audio-recorded interview, provision of verbal and written consent to participate, ability to read, understand and speak English, minimum age of 18 years and experience as one of the defined stakeholder groups.

\section{Data collection}

Individual interviews will be conducted by a graduate student under the supervision of experienced investigators. Individual interviews provide unique experiences that a participant may not be comfortable to build upon in a group setting, such as in a focus group. Following consent, participants will be given the option of filling in a demographics form. Additional medical details will be collected from patient charts after the interviews. By considering information such as gestational age, comorbidities, age, occupation, education and ethnicity, variation among interview groups will be accounted for in data analysis. Interviews will be recorded and transcribed with transcripts remaining free of identifying information. Data will be securely stored on a password-protected hospital hard drive.

\section{Interview guide and structure}

Interviews will be semistructured. A sample interview guide can be found in the online supplementary appendix B. Participants will be asked either in-person or over-thephone, what health outcomes they consider important in 
women with pregnancy-associated VTE, which outcomes should be reported in research within this area and why. If prompting is needed, then participants will be asked their opinion on currently reported outcomes that have been determined through the systematic review. Interviews will continue until no new outcomes are revealed within two successive sessions-referred to as 'saturation'. ${ }^{45}$

\section{Data analysis}

Audio recordings from participant interviews will be transcribed verbatim and written transcripts will be deidentified. The researcher will first review the transcripts to familiarise themselves with the data, noting initial ideas that represent outcomes. The transcripts will then be colour-coded line by line, grouping together related ideas into outcomes based on the taxonomy for outcomes in medical research, described earlier. ${ }^{41}$ All transcripts will be analysed by in duplicate by the researcher who conducted the interviews and an additional reviewer for a verification check. Discrepancies will be resolved by discussion with a third researcher. Comparisons within and across each interview will be conducted to determine factors responsible for variations in reported outcomes. Each outcome will be discussed and confirmed with at least two members of the research team until consensus is achieved. The list of outcomes from this stage will be combined with those determined through step I, the systematic review, and will constitute the long list of outcomes to inform step III, the Delphi survey.

\section{Step III: the Delphi method}

The Delphi method is designed to achieve convergence of opinion on the outcomes obtained through steps I and II in an organised, sequential manner. ${ }^{39} 46$

\section{Developing the survey}

Outcomes will then be categorised into medical domains following the same taxonomy as was used for the systematic review. ${ }^{41}$ Lay language descriptions will be provided for each outcome, determined and discussed by the research team and allied lay person representatives. The overarching aim of the survey is to allow participants to vote on which outcomes they believe should be included in the final COSPVenTE.

\section{Survey administration and participation}

The survey will be administered through Delphi Manager software, which allows global administration through an online, deidentified forum. By using the Delphi Survey Method, we aim to maximise its feasibility to include a wide representation of all stakeholder groups. In addition to patients and healthcare professionals who were represented in step II, we aim to have a representation of other stakeholders involved in the care of pregnant women with VTE, including patient representatives and general public with an interest in pregnancy-associated VTE, researchers, guideline developers and hospital administrators. In doing so, we aim to ensure that patients comprise at least $25 \%$ of all participants. We also hope to seek those involved in relevant organisations such as Thrombosis Canada, the International Society on Thrombosis and Haemostasis and the Thrombosis and Hemostasis Societies of North America and other parts of the world. To increase international representation, patients will be recruited through mother-baby blogs and patient advocacy groups in addition to the Hematologic Disorders in Pregnancy Clinic at Mount Sinai Hospital. Healthcare professionals and researchers will be recruited through professional contacts, as well as through author lists obtained from studies included in step I, the systematic review. The remaining stakeholder groups will be identified through published guidelines and known administrators of healthcare units. Once a participant has expressed interest in a study, they will be provided with a link to the survey via email, along with a description of the study and a consent form. Participants will also be asked to complete a demographic form, with a question asking which stakeholder group they identify with as compulsory to complete the survey.

\section{Delphi rounds}

Prior to the administration of the survey, participants will be provided with clear instructions and expectations regarding timelines on completing the survey. The survey will be open for 6 weeks per round, with bi-weekly emails reminding participants to complete it. The survey will consist of two rounds, both asking participants to rate identified outcomes on a scale of 1 (non-essential) to 9 (most essential). This 9-point Likert Scale based on the degree of importance is advocated by the Grading of Recommendations Assessment, Development and Evaluation working group. ${ }^{47}$ Scores $1-3$ will be regarded as 'nonessential', 4-6 as 'important but not critical' and 7-9 as 'critically important for inclusion'. Participants will have the option to select 'unable to score' should they lack the expertise to evaluate specific outcomes. During the second round, participants will receive their own scores as well as an anonymous, visual representation of how other groups of participants scored the same outcomes and will have the opportunity to alter or retain their scores based on the data presented. Any new outcomes emerging through the first round will also be incorporated into this round.

\section{Defining consensus}

To be eligible in the final COS for studies addressing pregnancy-associated VTE, outcomes must meet predefined criteria by the end of the second round. For consensus on inclusion, outcomes must be deemed 'critically important' indicated by a score of $7-9$ by $\geq 70 \%$ of participants and $1-3$ by $\leq 15 \%$ of participants. Outcomes ranked 'non-essential' indicated by a score of 1-3 by $>70 \%$ of participants and $7-9$ by $\leq 15 \%$ of participants will be excluded. 
Missing data and attrition

Participants will be given a 6-week window to complete each round of the survey, with clearly outlined expectations of timelines. Participants who complete partial responses in the first round will not be invited to the second round, this stipulation will be made clear in the original invitation to participate. Should the response rate not achieve $80 \%$, a level deemed acceptable by published recommendations, ${ }^{23}$ additional interventions will be implemented. Phone call and personal reminders as well as the extension of the survey deadlines may be used to improve the response rate. Any feedback after the first round regarding obstacles when completing the survey in its entirety will be considered before the second round.

\section{Step IV: consensus meeting}

At the end of the second round of the Delphi survey before participants submit their responses, they will be given a brief description of the next step of COS development, the in-person consensus meeting, and asked whether they would be willing to participate. Those interested will be invited via email with further instructions and details of the meeting. The aim is to ensure adequate representation of all stakeholder groups at the meeting.

The consensus meeting will occur in Toronto, Canada, in a conference-style format, using the nominal group technique (NGT) ${ }^{48}$ The COSPVenTE investigators, with the help of qualitative researchers will lead the meeting and moderate discussion. In NGT, participants are asked to first independently respond to questions posed by a moderator and then discuss answers within small groups. They are then asked to prioritise ideas or suggestions of all group members. NGT ensures that information is gathered from all members and then prioritises responses reflecting on the group's overall preferences. The meeting will begin with the moderator introducing outcomes that have not yet achieved consensus on their inclusion. Participants will have time to individually reflect and take notes, followed by discussion within small groups and the entire meeting. Participants will then independently, anonymously vote on outcomes through an electronic server. Following a break, participants will then be asked to follow a similar process for outcomes that have been included from step III of development. At this time, participants will be able to express any concerns or disagreements with outcomes that have been elected, but with emphasis on a final vote on outcomes, ranking them in order of priority. Although we will aim to include the least number of outcomes deemed critically important to form a 'minimum' set of outcomes, we intend to present, in addition to the 'core outcomes', the entire list of outcomes for mother and baby, stratified under the five outcome areas (mortality, clinical/physiological, life impact, resource use and adverse events), in descending order of importance as deemed by the Delphi process and consensus meeting. This will guarantee transparency and enable researchers to prioritise outcomes in addition to those considered 'core outcomes', representing all outcome areas, to ensure comprehensiveness of outcomes reporting. This will help maximise uptake in all future trials and studies addressing pregnancy-associated VTE. Additionally, we will ensure that no outcome deemed critically important by any stakeholder is removed in order to achieve a smaller list.

\section{Step V: determination of outcome definitions and measurements}

The COnsensus-based Standards for the selection of health Measurement INstruments method will be used to determine definitions and measurement tools for each outcome in the final COS for studies addressing pregnancy-associated VTE. Definitions and measurement tools will be evaluated based on validity, responsiveness, reliability and interpretability ${ }^{49}$ If necessary, an additional systematic review and/or Delphi survey may be conducted to help determine which definitions and measurement tools have been and should be used for the identified outcomes. ${ }^{50} \mathrm{~A}$ separate protocol for the conduct of this step will be developed and is beyond the scope of this publication.

\section{PATIENT AND PUBLIC INVOLVEMENT}

Although the steps of developing a COS as standardised, we will involve patients and relative stakeholders in steps II-IV, first through recruitment followed by interviews, the Delphi survey and a consensus meeting. The purpose of their involvement is to determine the health outcomes related to pregnancy-associated VTE most important to them. We have taken steps to ensure that the outcomes considered important by patients are represented in the final COS. We aim to involve patients in ensuring that the COS is disseminated widely through social media, in addition to ensuring knowledge translation to clinicians and researchers. Results of each step of COSPVenTE development will be published on the Outcome Reporting in Obstetric Studies website (https://www.obgyn.utoronto. $\mathrm{ca} /$ oros-project) and on the group's social media pages, enabling ongoing feedback from patients and the public.

\section{ETHICS AND DISSEMINATION}

This study, including consent forms for participation, has received approval from the Mount Sinai Hospital Research Ethics Board (REB 18-0314-E). We intend to publish separate papers for each stage of the project including the systematic review, patient interviews, and final COS consensus and development in open-access journals. All progress will be documented on the PROSPERO, COMET and CROWN databases. COSPVenTE findings will also be presented at national and international obstetrics, maternal-fetal medicine and haematology conferences. A timeline has been presented in the online supplementary appendix C.

\section{Author affiliations}

${ }^{1}$ Obstetrics \& Gynaecology, University of Toronto, Toronto, Ontario, Canada 
${ }^{2}$ Obstetrics \& Gynaecology, Maternal-Fetal Medicine, Mount Sinai Hospital, Toronto, Ontario, Canada

${ }^{3}$ Lunenfeld-Tanenbaum Research Institute, Mount Sinai Hospital, Toronto, Ontario, Canada

${ }^{4}$ McMaster University, Hamilton, Ontario, Canada

${ }^{5}$ Medicine and Laboratory Medicine and Pathobiology, University of Toronto, Toronto, Ontario, Canada

${ }^{6}$ Medicine, Division of Haematology, Mount Sinai Hospital, Toronto, Ontario, Canada

Twitter Rohan D'Souza @singingOB and Ann K Malinowski @SinaiHeme0B

Contributors RD'S and AKM: conceived the idea. AK: drafted the protocol manuscript with the assistance and revision of LT based on COMET handbook guidelines and other relevant publications. RD'S: AK's primary thesis supervisor is the founder and lead investigator on the OROS project, with experience in developing COS for obstetric conditions; he will supervise all the steps of COS development. AKM (maternal-fetal medicine) and NS (haematology): are the content experts on pregnancy-associated VTE. All authors reviewed the manuscript at least once and approved the final version.

Funding This project is funded in part by the Frederick Banting and Charles Best Canadian Graduate Scholarship-Master's Award (CGS M) for May 2019-May 2020 received by Alexandria King.

Competing interests RD'S has received speaking honoraria from Ferring, Canada for presentations unrelated to this topic. NS has received speaking honoraria from Sanofi.

Patient consent for publication Not required.

Provenance and peer review Not commissioned; externally peer reviewed.

Open access This is an open access article distributed in accordance with the Creative Commons Attribution Non Commercial (CC BY-NC 4.0) license, which permits others to distribute, remix, adapt, build upon this work non-commercially, and license their derivative works on different terms, provided the original work is properly cited, appropriate credit is given, any changes made indicated, and the use is non-commercial. See: http://creativecommons.org/licenses/by-nc/4.0/.

\section{ORCID iDs}

Rohan D'Souza http://orcid.org/0000-0002-4049-2017

Nadine Shehata http://orcid.org/0000-0002-9267-6256

\section{REFERENCES}

1 Devis $P$, Knuttinen MG. Deep venous thrombosis in pregnancy: incidence, pathogenesis and endovascular management. Cardiovasc Diagn Ther 2017;7:S309-19.

2 Liu S, Rouleau J, Joseph KS, et al. Epidemiology of pregnancyassociated venous thromboembolism: a population-based study in Canada. J Obstet Gynaecol Can 2009;31:611-20.

3 CDC. Pregnancy mortality surveillance system. In: Atlanta: centers for disease control and prevention, 2019.

4 Turner LA, Cyr M, Kinch RAH, et al. Under-reporting of maternal mortality in Canada: a question of definition. Chronic Dis Can 2002;23:22-30.

5 Wik HS, Jacobsen AF, Sandvik L, et al. Long-term impact of pregnancy-related venous thrombosis on quality-of-life, genera health and functioning: results of a cross-sectional, case-control study. BMJ open 2012;2:e002048.

6 Kahn SR. The post-thrombotic syndrome. Hematology Am Soc Hematol Educ Program 2016;2016:413-8.

7 Kahn SR, Comerota AJ, Cushman M, et al. The postthrombotic syndrome: evidence-based prevention, diagnosis, and treatment strategies: a scientific statement from the American heart association. Circulation 2014;130:1636-61.

8 Appelen D, van Loo E, Prins MH, et al. Compression therapy for prevention of post-thrombotic syndrome. Cochrane Database Syst Rev 2017;9:CD004174.

9 Skervin AL, Thapar A, Franchini AJ, et al. Systematic review and meta-analysis of utility of graduated compression stockings in prevention of post-thrombotic syndrome. Eur J Vasc Endovasc Surg 2016;51:838-45.

10 Wik HS, Jacobsen AF, Sandvik L, et al. Prevalence and predictors for post-thrombotic syndrome 3 to 16 years after pregnancy-related venous thrombosis: a population-based, cross-sectional, casecontrol study. J Thromb Haemost 2012;10:840-7.
11 American College of Obstetricians and Gynecologists Women's Health Care Physicians. ACOG practice bulletin No. 138: inherited thrombophilias in pregnancy. Obstet Gynecol 2013;122:706-17.

12 Bates SM, Middeldorp S, Rodger M, et al. Guidance for the treatment and prevention of obstetric-associated venous thromboembolism. J Thromb Thrombolysis 2016;41:92-128.

13 Association $\mathrm{CH}$. Consensus statement on the care of patients with sickle cell disease in Canada 2015. Available: http://sicklecellanemia. ca/pdf_2016/CANHAEM.pdf

14 Nelson-Piercy CMP, Mackillop L. RCOG green-top guideline No. 37A: reducing the risk of venous thromboembolism during pregnancy and the puerperium, 2015. Available: https://www.rcog.org.uk/ globalassets/documents/guidelines/gtg-37a.pdf

15 Malinowski AK, Shehata N, D'Souza R, et al. Prophylactic transfusion for pregnant women with sickle cell disease: a systematic review and meta-analysis. Blood 2015;126:2424-35.

16 Chess LE, Gagnier JJ. Applicable or non-applicable: investigations of clinical heterogeneity in systematic reviews. BMC Med Res Methodol 2016;16:19.

17 Malinowski AK, D'Souza R, Khan KS, et al. Reported outcomes in perinatal iron deficiency anemia trials: a systematic review. Gynecol Obstet Invest 2019;84:417-34.

18 Koot MH, Boelig RC, Van't Hooft J, et al. Variation in hyperemesis gravidarum definition and outcome reporting in randomised clinical trials: a systematic review. BJOG 2018;125:1514-21.

19 Duffy J, Hirsch M, Kawsar A, et al. Outcome reporting across randomised controlled trials evaluating therapeutic interventions for pre-eclampsia. BJOG 2017;124:1829-39.

20 Rogozinska E, Fen Y, Molyneaux E, et al. Variation in outcomes in trials reporting effects of diet and lifestyle based intervention on pregnancy outcomes: a systematic review. Pregnancy Hypertens 2014;4:237

21 Al Wattar $\mathrm{BH}$, Placzek $\mathrm{A}$, Troko J, et al. Variation in the reporting of outcomes among pregnant women with epilepsy: a systematic review. Eur J Obstet Gynecol Reprod Biol 2015;195:193-9.

22 Viau-Lapointe J, D'Souza R, Rose L, et al. Development of a core outcome set for research on critically ill obstetric patients: a study protocol. Obstet Med 2018;11:132-6.

23 Williamson PR, Altman DG, Bagley $\mathrm{H}$, et al. The COMET handbook: version 1.0. Trials 2017;18:280.

24 Core Outcome Measures in Effectiveness Trials (COMET) Initiative. COS uptake, 2018. Available: http://www.comet-initiative.org/ cosuptake [Accessed 30 Aug 2018].

25 Khan K. The CROWN initiative: Journal editors invite researchers to develop core outcomes in women's health. BJOG 2014;121:1181-2.

26 CROWN Initiative. Core outcomes in women's and newborn health Available: http://www.crown-initiative.org/14-2/about/

27 Moss N, Daru J, Lanz D, et al. Involving pregnant women, mothers and members of the public to improve the quality of women's health research. BJOG 2017;124:362-5.

28 D'Souza R, Shah PS, Sander B. Clinical decision analysis in perinatology. Acta Obstet Gynecol Scand 2018;97:491-9.

29 D'Souza R, Tomlinson G, McKelvey K, et al. Preferences of pregnant women and family members for combined maternal-fetal health states arising from the use of anticoagulation in pregnancy. Vancouver, BC: Medical Decision Making, 2017: PS2-15.

30 Rogozińska E, Eckert LO, Khan KS. Reducing research waste through the standardisation of outcomes and definitions. BJOG 2019;126:308-309.

31 Dadouch R, Faheim M, Juando-Prats C, et al. Development of a core outcome set for studies on obesity in pregnant patients (COSSOPP): a study protocol. Trials 2018;19:655.

32 Kirkham JJ, Davis K, Altman DG, et al. Core outcome Set-STAndards for development: the COS-STAD recommendations. PLoS Med 2017; $14:$ e1002447

33 Kirkham JJ, Gorst S, Altman DG, et al. Core outcome SetSTAndardised protocol items: the COS-STAP statement. Trials 2019;20:116.

34 Malinowski A, Shehata N, D'Souza R. Core outcome set for perinatal venous thromboembolism, 2017. Available: http://www.cometinitiative.org/studies/details/839?result=true

35 Liberati A, Altman DG, Tetzlaff J, et al. The PRISMA statement for reporting systematic reviews and meta-analyses of studies that evaluate health care interventions: explanation and elaboration. $J$ Clin Epidemiol 2009;62:e1-34.

36 King A, Malinowski AK, D'Souza R. Systematic review of reported outcomes in studies addressing perinatal venous thromboembolism: PROSPERO Registration, 2019. Available: https://www.crd.york.ac. uk/prospero/display record.php?ID=CRD42019111479 [Accessed 27 May 2020]. 
37 McCoy CE. Understanding the use of composite endpoints in clinical trials. West J Emerg Med 2018;19:631-4.

38 Meher S, Alfirevic Z. Choice of primary outcomes in randomised trials and systematic reviews evaluating interventions for preterm birth prevention: a systematic review. BJOG 2014;121:1188-94.

39 Duffy J, Rolph R, Gale C, et al. Core outcome sets in women's and newborn health: a systematic review. BJOG 2017;124:1481-9.

40 Smith PP, Dhillon-Smith RK, O'Toole E, et al. Outcomes in prevention and management of miscarriage trials: a systematic review. BJOG 2019;126:176-89.

41 Dodd S, Clarke M, Becker L, et al. A taxonomy has been developed for outcomes in medical research to help improve knowledge discovery. J Clin Epidemiol 2018;96:84-92.

42 Cypress BS. Qualitative research: the "what," "why," "who," and "how"! Dimens Crit Care Nurs 2015;34:356-61.

43 Thirsk LM, Clark AM. Using qualitative research for complex interventions. Int J Qual Methods 2017;16:160940691772106.

44 Palinkas LA, Horwitz SM, Green CA, et al. Purposeful sampling for qualitative data collection and analysis in mixed method implementation research. Adm Policy Ment Health 2015;42:533-44.
45 Green J, Thorogood N. Qualitative methods for health research. 3 edn. SAGE Publications, 2014.

46 Sinha IP, Smyth RL, Williamson PR. Using the Delphi technique to determine which outcomes to measure in clinical trials: recommendations for the future based on a systematic review of existing studies. PLoS Med 2011;8:e1000393.

47 Guyatt GH, Oxman AD, Kunz R, et al. Grade guidelines: 2. framing the question and deciding on important outcomes. J Clin Epidemiol 2011;64:395-400.

48 CDC, Department of Health and Human Services. Gaining consensus among stakeholders through the nominal group technique. evaluation briefs, 2006. Available: https://www.cdc.gov/healthyyouth/ evaluation/pdf/brief7.pdf

49 Mokkink LB, Terwee CB, Patrick DL, et al. The COSMIN checklist for assessing the methodological quality of studies on measurement properties of health status measurement instruments: an international Delphi study. Qual Life Res 2010;19:539-49.

50 Prinsen CAC, Vohra S, Rose MR, et al. How to select outcome measurement instruments for outcomes included in a "Core Outcome Set" - a practical guideline. Trials 2016;17:449. 